(C2020, Elsevier. Licensed under the Creative Commons Attribution-NonCommercialNoDerivatives 4.0 International http://creativecommons.org/about/downloads 


\title{
Cosm-nutraceutical nanovesicles for acne treatment: Physicochemical characterization and exploratory clinical experimentation
}

\author{
Sandra Sherif Amer ${ }^{1,2,3}$, Maha Nasr ${ }^{4 *}$, Rasha T.A. Abdel-Aziz ${ }^{5}$, Noha H. Moftah ${ }^{5}$, Amr El \\ Shaer $^{2}$, Elena Polycarpou ${ }^{2}$, Wael Mamdouh ${ }^{3}$, Omaima Sammour ${ }^{4}$ \\ ${ }^{1}$ Department of Pharmaceutics, Faculty of Pharmacy and Drug Manufacturing, Pharos University \\ in Alexandria, Alexandria, Egypt \\ ${ }^{2}$ Drug Discovery, Delivery and Patient Care (DDDPC) Theme, School of Life Sciences, Pharmacy \\ and Chemistry, Department of Pharmacy, Kingston University London, Kingston upon Thames, \\ Surrey, KT1 2EE \\ ${ }^{3}$ Department of Chemistry, School of Sciences and Engineering, The American University in \\ Cairo, Egypt \\ ${ }^{4}$ Department of Pharmaceutics and Industrial Pharmacy, Faculty of Pharmacy, Ain Shams \\ University, Cairo, Egypt \\ ${ }^{5}$ Department of Dermatology, STD's and Andrology, Faculty of Medicine, Minia University, Al \\ Minya, Egypt
}

Correspondence to: Maha Nasr, $\mathrm{PhD}$

Email: drmahanasr@pharma.asu.edu.eg

Cellular phone number: +201003450279

Fax: 002024051107

Postal Address: Ain Shams University- Faculty of Pharmacy- Department of Pharmaceutics and Industrial Pharmacy- Monazamet El Wehda El Afrikia St., El Abbassia, Cairo (Egypt) 


\begin{abstract}
$\underline{\text { Abstract }}$
The full exploration of the 'nutraceuticals' therapeutic potential in cosmetics has been hindered by their poor stratum corneum permeation. Therefore, the aim of the present study was to formulate a nutraceutical; quercetin, in novel vitamin $\mathrm{C}$ based nanovesicles (aspasomes), and to explore their beneficial effects in the treatment of acne. Aspasomes were characterized for their particle size, zeta potential, entrapment efficiency (EE\%), 3-months storage stability, skin deposition/permeation, antioxidant potential, and morphology. Aspasomes antibacterial efficacy on Propionibacterium acnes using the zone of inhibition assay was also tested, whilst their safety on skin fibroblastic cells was assessed in vitro using 3T3 CCL92 cell lines. An exploratory clinical trial was conducted in acne patients, and the percentage reduction of inflammatory, noninflammatory and total acne lesions was taken as the evaluation criterion. Results revealed that quercetin-loaded aspasomes displayed a desirable nanometer size (125-184 nm), negative charge with good storage stability, and high skin deposition reaching 40\%. Aspasomes managed to preserve the antioxidant activity of quercetin, and exhibited a significantly higher antibacterial effect $(15 \pm 1.53 \mathrm{~mm})$ against Propionibacterium acnes than quercetin alone $(8.25 \pm 2.08 \mathrm{~mm})$, and were safe on skin fibroblastic cells. Upon clinical examination in 20 acne patients (14 females, 6 males), quercetin aspasomes exhibited reduction percentages of $77.9 \%, 11.8 \%$ and $55.3 \%$ for inflammatory lesions, comedones and total lesions respectively. This opens vast applications of the presented formulation in the treatment of other oxidative skin diseases, and delineates the nutraceuticals and nanoformulations prepared from natural materials as promising dermatological treatment modes.
\end{abstract}

Keywords: Vitamin C; Aspasomes; Acne; Quercetin; Nanoparticles; Antibacterial. 


\section{Introduction}

From the plethora of recent research, many novel nanosystems with topical delivery benefits were discovered (Bseiso et al., 2015; Abdelgawad et al., 2017; Fadel et al., 2017; Hatem et al., 2018a,b; Amer et al., 2019; El-Kayal et al., 2019; Elmowafy et al., 2019; Shaaban et al., 2019), with the ability to improve the ex-vivo cutaneous drug deposition and permeation. An antioxidant nanovesicular system; aspasomes was first reported by Gopinath et al., 2004, but was only recently assessed for their dermatological application in the treatment of androgenic alopecia by Hatem et al., 2018c. The basic component of aspasomes responsible for the anti-oxidant effect is ascorbyl palmitate, which is the lipid soluble and more stable form of ascorbic acid (vitamin C) (Gopinath et al.,2004; Saini et al., 2014).

A dermatological disease which could benefit from the antioxidant properties of an aspasomal delivery system is acne (Morris, 1954; Peirce et al., 1959). Acne vulgaris is a chronic dermatological disease of the pilosebaceous gland (hair follicles associated with the oil glands) affecting people in their adolescence (Strauss et alo, 2007; Titus and Hodge, 2012). It is marked by the presence of different forms of acne lesions, appearing as inflammatory pimples (whitehead \& blackhead), papules, pustules, cysts, and nodules, and non-inflammatory lesions (comedones) (Adityan et al., 2009; Fabbrocini et al., 2010; Najafi-Taher and Amani, 2017). The main causative organism for acne is Propionibacterium acnes (P. acnes) residing in the sebaceous glands. Together with the excess sebum present in the skin, they result in the blockage of pores, and the greasy look of the skin in acne patients. The antioxidant nature of aspasomes mainly attributed to its content of ascorbyl palmitate would help to overcome the implicated action of reactive oxygen species and lipid peroxide in the pathogenesis and progression of acne (Mills et al., 2016). 
Owing to the reported therapeutic power of nutraceuticals, an antioxidant molecule; quercetin was chosen in the current study as the therapeutic moiety of choice to be loaded within aspasomes, owing to its anti-inflammatory, anti-bacterial and anti-oxidant properties (Maciel et al., 2013). To further accentuate the cosmetic nature of the prepared aspasomes, several essential oils namely tea tree and neem oils were tested for inclusion in the aspasomal formulations, owing to their favorable contribution in acne treatment (Raman et al., 1995; Enshaieh et al., 2007; Ulbricht et al., 2011; Vijayan et al., 2013). The present manuscript represents the first attempt for the clinical use of aspasomes in acne treatment. The prepared aspasomes were characterized for their pharmaceutical properties such as size, charge, storage stability, skin deposition ability, antioxidant potential, in addition to their safety and their antibacterial activity against $P$. acnes. Furthermore, an exploratory clinical trial was conducted to assess their therapeutic potential in the treatment of acne.

\section{Experimental Section}

\subsection{Materials}

Quercetin, ascorbyl palmitate, cholesterol, diphenyl-1-picrylhydrazyl (DPPH) and dicetyl phosphate were all purchased from Sigma Aldrich, Germany. Tea tree oil was purchased from Alpha Pharmaceutical Co., Egypt. Neem oil was purchased from Chemajet chemical Co., Egypt. Propionibacterium acnes ATCC 6919, methanol, acetonitrile and water (HPLC analytical grade solvents), and sodium chloride were purchased from Fisher Scientific, UK. Disodium hydrogen phosphate, sodium chloride and potassium dihydrogen phosphate were purchased from El Nasr chemical Co., Egypt. Acetyl Uranyl (Uranyl acetate -2- hydrate) was purchased from Allied signal, Riedel- dehaen, Germany. Horse blood agar, 3T3 CCL92 skin fibroblasts, trypsin, penicillin, streptomycin, barium chloride dehydrate, sulfuric acid, hydrogen peroxide, neutral red, ethanol 
HPLC analytical grade and chloroform were all purchased from Sigma Aldrich Co., UK. Dulbecco Modified Eagle's Medium (DMEM), and FBS (Fetal Bovine serum 10\%) were purchased from Gibco by Life Technologies, Thermo Fisher Scientific, USA. Mueller Hinton agar, brain heart infusion, and the anaerobic indicator were purchased from Thermo Scientific ${ }^{\mathrm{TM}} \mathrm{Oxoid}^{\mathrm{TM}}$ Anaerogen ${ }^{\mathrm{TM}}$, UK. Industrial methylated spirit $70 \%$ (IMS) was purchased from Atom Scientific, UK. Panthenol ${ }^{\circledR}$ cream was purchased from The Nile Co. for Pharmaceuticals and Chemical Industries, Egypt. Viva-spin tubes (PES membrane, MWCO 300 $\mathrm{kDa}$ ) were purchased from Sartorius, Netherlands.

\subsection{Methods}

\subsubsection{Preparation of quercetin aspasomes using the thin film hydration method}

Aspasomes were prepared using the thin film hydration technique, in which 30, 56.6 and $13.5 \mathrm{mg}$ of ascorbyl palmitate, cholesterol and dicetyl phosphate respectively, $10 \mathrm{mg}$ quercetin and $10 \mu \mathrm{L}$ of either tea tree oil, neem oil or both were used to formulate three different aspasomal vesicles, as shown in Table 1. The amounts were selected based on a preliminary study (data not shown).

The lipidic ingredients were dissolved in organic solvent mixture of chloroform: methanol (2:1 v/v) (Nasr et al., 2008; Aref at al., 2017; Agiba et al., 2018). The solvent mixture was then allowed to evaporate at $40^{\circ} \mathrm{C}$ and $150 \mathrm{rpm}$ (BUCHI Rotavapor R-114 WB B-480, USA) until the formation of a thin film of dry lipid. This was followed by portion-wise rehydration with $10 \mathrm{~mL}$ phosphate buffered saline (PBS, $\mathrm{pH}$ 7.4). The flask was rotated for 60 minutes at constant temperature allowing the hydration and maturation of the vesicles. The hydrated liquid aspasomal formulations were stored in glass vials at $4-8^{\circ} \mathrm{C}$ for further investigations.

\subsubsection{Determination of the particle size and zeta potential of the aspasomal formulations}


The particle size and zeta potential of the freshly prepared aspasomal formulations were determined using the Zeta-sizer (ZS90, Malvern Instruments, Ltd., Worcestershire, UK). Samples were appropriately diluted with filtered deionized water using a disposable cuvette at $25^{\circ} \mathrm{C}$, detection angle $173^{\circ}$, following 2-minutes equilibration (Bseiso et al., 2016; Mouez et al., 2016; Fadel et al., 2018).

\subsubsection{Determination of quercetin entrapment efficiency (EE\%) in aspasomes}

The entrapped amount of quercetin loaded in aspasomal formulations was determined by an ultra-filtration method using viva-spin centrifugal tubes, where the aspasomal formulations (2 $\mathrm{mL}$ ) were transferred to the upper chamber of the viva-spin tubes and centrifuged at $4000 \mathrm{rpm}$ at $25^{\circ} \mathrm{C}$ for 30 minutes (Bench Biofuge Centrifuge, Heraeus, Germany). An aliquot of the filtrate was then diluted using methanol (Aref et al., 2017; Du et al., 2017), and the amount of the unentrapped (free) quercetin was then analyzed using HPLC (Dionex Ultimate 3000, USA), utilizing a mobile phase acetonitrile:water (40:60) flowing at $1 \mathrm{~mL} / \mathrm{min}$ through a C18 HPLC column (Thermo scientific, BDS Hypersil column, $4.6 \times 250 \mathrm{~mm}, 5 \mu \mathrm{m}$, USA), and the effluent was analyzed at $354 \mathrm{~nm}$ (Kumari et al., 2010).

The EE\% was calculated according to equation 1:

$\mathrm{EE} \%=\frac{\text { Initial Quercetin amount-free unentrapped quercetin }}{\text { Initial quercetin }} \times 100 \quad$ (Equation 1)

\subsubsection{Physical stability assay of aspasomes}

The aspasomal formulations were assessed for their physical stability, where samples were re-examined for changes occurring in the particle size, zeta potential and EE\% after storage in the refrigerator at a temperature of $4-8^{\circ} \mathrm{C}$ for a period of three months. Samples were centrifuged first then assessed (Nasr et al., 2008; Aref at al., 2017). 


\subsubsection{Ex-vivo skin deposition of quercetin aspasomes}

The ex-vivo skin deposition of quercetin selected aspasomal formulations was performed using the Franz diffusion apparatus (Variomag Telesystem, Germany) and analyzed by HPLC as described above. On the day of the experiment, the excised rat skin was defrosted, moistened with PBS of pH 7.4 one hour before usage, cut into small square pieces, and mounted between the receptor and the donor compartments of cells $\left(1.77 \mathrm{~cm}^{2}\right)$ allowing the stratum corneum to face upwards (Singh et al., 2014; Bsieso et al., 2015; Nasr and Abdel-Hamid, 2016; Nasr et al., 2017). A specific amount of the aspasomes $(0.1 \mathrm{~mL})$ was placed onto the skin in the donor compartment, and the receptor compartment was filled with $7.5 \mathrm{~mL}$ PBS of pH 7.4 stirred at 150 rpm at $37^{\circ} \mathrm{C}$ (Gopinath et al., 2004; El Zaafarany et al., 2010). Five hundred microliter samples were withdrawn from the receptor compartments after 6 hours, diluted with methanol and analyzed for the permeated quercetin using HPLC as previously described. The skin samples were removed, washed five times with distilled water, then wiped with a clean tissue and dried by a clean filter paper to remove any excess aspasomal formulation, and finally placed in vials containing $10 \mathrm{~mL}$ methanol. Vials were then placed in the bath sonicator (Julabo, USR 3, Germany) for a period of one hour to allow extraction of the deposited quercetin. The amount of quercetin accumulated into the skin was expressed as a percentage of the total amount applied on the skin.

\subsubsection{Diphenyl-1-picrylhydrazyl (DPPH) anti-oxidant assay of selected quercetin aspasomal formulations}

The anti-oxidant activity of the selected quercetin aspasomal formulation was determined using the DPPH free radical scavenging assay. DPPH exhibits an obvious absorption maximum at $515 \mathrm{~nm}$ giving a characteristic purple color. The anti-oxidant potential is directly proportional to the disappearance of the purple color of the DPPH, and the formation of the yellow color of the 
reduced DPPH, which depends on the number of hydrogen atoms donated by the anti-oxidant substance, and hence absorbed by the DPPH. Therefore, the anti-oxidant activity can be examined by following the decrease of UV absorption at $515 \mathrm{~nm}$ (Milton Roy, USA)(Ashraf et al., 2018). A $5 \mu \mathrm{L}$ aliquot of quercetin, quercetin aspasomal formulations, and ascorbic acid control in methanol were added to $200 \mu \mathrm{L}$ of DPPH methanolic solution of concentration $0.004 \% \mathrm{w} / \mathrm{v}$. Absorbance readings of DPPH tested in 96 well plates were recorded immediately at $515 \mathrm{~nm}$. The $\mathrm{IC}_{50}$ values were estimated and the calculation of percentage inhibition of DPPH radical was calculated as previously described (Ashraf et alo, 2018).

\subsubsection{Morphological characterization of the selected quercetin aspasomal formulation using transmission electron microscope (TEM)}

The selected aspasomal formulation was morphologically examined by the TEM (JEM-100S, Joel, Japan) after negative staining (Nasr et al., 2013). One drop of diluted sample was placed on a 200-mesh copper grid, then one drop of $1 \%$ uranyl acetate stain was added and allowed to dry and the excess fluid was removed before examination.

\subsubsection{Assessment of the antibacterial potency of quercetin aspasomes}

P. acnes was cultured under anaerobic conditions (a gas mixture of $80 \%$ nitrogen, $10 \%$ carbon dioxide and $10 \%$ hydrogen with no oxygen) at a temperature of $37^{\circ} \mathrm{C}$ (Kishishita et al., 1980; Bojar and Holland, 2004; Perry and Lambert, 2006). The bacteria were hydrated by brain heart infusion (BHI) and thoroughly mixed (Tally et al., 1978; Kumar et al., 2007), then they were inoculated onto a highly enriched blood agar prepared from Mueller-Hinton (MH) agar base and horse blood (Dali et al., 2001). The agar plates were placed into a jar where a gas tea bag and an anaerobic indicator were added to ensure that the conditions were free of oxygen (Kumar et al., 2007). The gas jar was then placed in the incubator and checked for bacterial growth on a two- 
day basis for a period of 5-7 days. Following bacterial growth, a cotton swab of the bacteria was placed in PBS and vortexed several times allowing it to be the same color as 0.5 McFarland standard (equivalent to $1.5 * 10^{8} \mathrm{CFU} / \mathrm{mL}$ of micro-organisms) (Wulansari et al., 2017). Afterwards, a swab was taken from this solution to cover the blood agar plates which were then left to settle for a minute. Ten microliter samples of ethanol (as a control), quercetin ethanolic solution, and the selected aspasomal formulation were then added on to the agar plates (Wulansari et al., 2017). The plates were then covered and left in a well-sealed gas jar containing a tea bag, and an indicator to ensure that the conditions were free of oxygen. The jar was then placed in the incubator for 2 days after which the plates were removed, and the inhibition zones were measured in $\mathrm{mm}$.

\subsubsection{Safety of quercetin aspasomes on skin fibroblasts}

The study was carried on 3T3 CCL92 cells, also known as the skin fibroblasts 3T3-Swiss Albino mouse (ATCC ${ }^{\circledR}$ CCL92 ${ }^{\mathrm{TM}}$ ) to test the topical safety of the selected aspasomal formulation, similar to what was reported with other authors (Jírová et al., 2003; Calabro et al., 2008). The experiment was carried out in an aseptic environment inside the tissue culture hood. The nutrient media used was Dulbecco modified eagle's media (DMEM), to which $10 \%$ fetal bovine serum (FBS) and 1\% penicillin-streptomycin antibiotics were added (Hockley and Baxter, 1986; Calabro et al., 2008). The vial containing the cells was removed from the liquid nitrogen where it was stored, defrosted and thawed in the water bath $\left(37^{\circ} \mathrm{C}\right)$, then it was sterilized before being placed in the hood. The desired volume was withdrawn and placed in three $75-\mathrm{mL}$ flasks each containing previously prepared DMEM. Flasks were then placed in the incubator, where cells were checked after two days for viability, motility and confluency. After reaching half-confluency, they were incubated again for another two days. The cells were afterwards rinsed with $0.25 \%(\mathrm{w} / \mathrm{v})$ 
trypsin, then 3-mL trypsin were added to the flask, agitated gently, and incubated for 3-5 minutes to activate the cells and detach them. Afterwards, $7-\mathrm{mL}$ nutrient medium were added to the flask to inactivate the trypsin, then all the $10 \mathrm{~mL}$ were placed in a centrifuge tube and centrifuged at a rate of $125 \mathrm{x} \mathrm{g}$ for 10 minutes to produce a pellet that was used for further sub-culturing. After reaching a cell count of $5 \times 10^{4}$ cells $/ \mathrm{mL}$, cells were placed in the wells. Samples tested were the selected aspasomal formulation, the quercetin solution, in addition to the negative control where nutrient media was added to cells, as well as the hydrogen peroxide which was used as a positive control. The plates were then covered and incubated at adequate conditions $\left(37^{\circ} \mathrm{C} / 5 \% \mathrm{CO} 2\right.$ in air atmosphere/humidified atmosphere) overnight in the incucyte (Thermo Fischer Scientific, USA). The neutral red dye solution at a concentration of $40 \mu \mathrm{g} / \mathrm{mL}$ was prepared and incubated overnight in the same conditions as the cells. After 24 hours, plates were checked under the microscope (Olympus CX41-MET, UK) for their viability and morphological changes. Samples were then decanted, and nutrient media containing neutral red dye were added to the cells followed by incubation for another two hours. A de-staining solution was then added to the plates, and plates were then shaken in the microtiter plate shaker for 10 minutes, followed by extraction of the neutral red, and the optical density (OD) of the neutral red extract was measured at $540 \mathrm{~nm}$ using a spectrophotometer (EnSpire Alpha plate reader, Perkin Elmer, USA)(Lasarow et al., 1992; Repetto et al., 2008; Ates et al., 2017).

\subsubsection{Clinical evaluation of quercetin aspasomes on acne patients}

The clinical study included patients of both genders suffering from acne vulgaris, selected from the Dermatology Out-patient Clinic of Minia University Hospital, Al-Minya, Egypt. This study included patients suffering from mild or moderate acne vulgaris according to grading of NilFroushzadeh et al., 2009. The study included 20 patients suffering from acne vulgaris (6 males 
and 14 females), their age ranged from 14 to 27 years, of acne lesions duration between 1 to 7 months. Inclusion criteria involved patients who did not receive any treatment for acne or oral contraceptive pills at least 3 months prior to the study. Exclusion criteria included pregnant and nursing patients or those who suffered from hyperandrogenism. The clinical trial has been carried out in accordance with "The Code of Ethics of the World Medical Association (Declaration of Helsinki). An informed consent was obtained from each patient or his guardians, and the study was approved by the Committee for Postgraduate Studies and Research of Minia University, and the Committee for Postgraduate Studies and Research of Ain Shams University (REC- ASU\#31). Patients were instructed to apply a thin film of the selected quercetin aspasomal formulation (a volume of $100 \mu \mathrm{L}$ corresponding to a quercetin dose of $100 \mu \mathrm{g}$ ) on the right side of the face once daily and a placebo formulation (Panthenol ${ }^{\circledR}$ ) on the left side of the face once daily for 12 hours. Panthenol ${ }^{\circledR}$ was chosen as a negative control, since there were no topical products available in the market for quercetin. The treatment period continued up to 8 weeks, and patients were instructed to report any discomfort or irritation encountered during the study (Nasr et al., 2018). Patients were photographed every 2 weeks and evaluated clinically after 8 weeks on both sides of the face through the counting of comedones, inflammatory and total acne lesions by 2-blinded dermatologists. The percentage reduction was determined for each of the inflammatory, comedonal, and the total acne lesions where it was calculated by the following equation (Barakat et al., 2017):

Number of lesions before administration-Number of lesions after administration Number of lesions before administration

x 100\% (Equation 2)

\subsubsection{Statistical analysis}


Experiments were done in triplicate and expressed as mean \pm standard deviation (S.D). A comparison using one-way analysis of variance (ANOVA) was carried out, followed by Tukey Kramer post-test or paired t-test using Graphpad ${ }^{\circledR}$ Instat software. A difference between means was considered significant if the $\mathrm{P}$ value was less than or equals to 0.05 .

\section{Results and Discussion}

\subsection{Preparation of quercetin aspasomal formulations}

Till current date, only few papers about aspasomes have been published. Aspasomes formation required the three basic ingredients; ascorbyl palmitate, cholesterol and dicetyl phosphate. Owing to the bilayer forming property of ascorbyl palmitate, it is able to form vesicles (aspasomes) which exhibit an anti-oxidant action as reported by Gopinath et al., 2004, with the aid of cholesterol and dicetyl phosphate. Some concerns were raised by Meves et al., 2002 that ascorbyl palmitate might cause skin damage after exposure to UV radiation, however this study was conducted in vitro in keratinocytes, and was not verified further by in vivo or clinical studies.

The three ingredients (ascorbyl palmitate, cholesterol and dicteyl phosphate) are considered essential for the formation of stable vesicles, where cholesterol was reported to modify the membrane properties and increase its mechanical strength (Kirby et al., 1980; Magarkar et al., 2014). As reported by Kirby et al., 1980; Lee et al., 2005; Magarkar et al., 2014, cholesterol intercalates in the bilayer with its polar head, and due to its hydrophobic properties, it occupies the interior portion of lipid bilayers and is able to fill the gaps and restrict any membrane component movements. Furthermore, a study reported by Owen et al., 2018 suggested that cholesterol is important as it protects the lipid bilayer from hydrolytic degradation, oxidative stress, and water injury. The third ingredient is the dicetyl phosphate which was included as a negative charge 
inducer to create vesicles with stronger interaction with the surface of the skin achieving better topical delivery (Chen et al., 2012), and creates an electrostatic repulsion between adjacent vesicles, hence delaying the formation of aggregates.

\subsection{Determination of the particle size and zeta potential of the aspasomal formulations}

As shown in Table 1, quercetin aspasomal formulations displayed a particle size range of 125-184 nm with no separated oil droplets, suggesting the successful intercalation of oils within the bilayer. F3 formulated with $20 \mu \mathrm{L}$ total oil content displayed significantly lower particle size compared to F1 and F2 $(\mathrm{P}<0.05)$, which may be attributed to the double fluidizing ability of the oils on the lipidic ascorbyl palmitate (Herman and Herman, 2014). Zeta potential measurement showed that all formulations were negatively charged with no significant difference between them $(\mathrm{P}>0.05)$, which is attributed to the presence of the negative charge inducer dicetyl phosphate (Villasmil-Sánchez et al., 2010; Chibowski andSzczes, 2016).

\subsection{Determination of quercetin $\mathrm{EE} \%$ in aspasomes}

As shown in Table 1, all formulations displayed high EE\% for quercetin ranging from (96$100 \%$ ). It was also shown that formulae F2 and F3 prepared using tea tree oil showed a significantly higher $\mathrm{EE} \%$ for quercetin than $\mathrm{F} 1$ prepared using neem oil only $(\mathrm{P}<0.05)$, suggesting augmented solubilization of quercetin in tea tree oil, with consequent increase in its entrapment within the bilayers. The high EE\% encountered for all formulations could be ascribed to the utilization of the thin film hydration technique which creates multilamellar vesicles capable of entrapping large amounts of hydrophobic drugs as quercetin (Sharma and Sharma, 1997; Morilla et al., 2002; Sinico et al., 2005). Furthermore, the high EE\% may be attributed to the lipophilicity of the quercetin $(\log \mathrm{P}=1.82 \pm 0.32)$ (Rothwell et al., 2005), enabling its subtle 
incorporation within the hydrophobic ascorbyl palmitate bilayer membrane. Furthermore, the presence of cholesterol in the aspasomal composition increases the hydrophobicity of the bilayer, therefore prompting more drug entrapment within the bilayers and less drug leakage, thus allowing a high EE\% (Lee et al., 2005; Nasr et al., 2008; Briuglia et al., 2015). Also, it was reported by Moribe et al., 1999 and Singh et al., 2014 that cholesterol increases the micro-viscosity of the bilayer, resulting in an increase in its stability, and a decrease in its permeability, and therefore causing higher drug loading, entrapment, and retention. Since formulations F2 and F3 displayed higher $\mathrm{EE} \%$ than $\mathrm{F} 1$, they proceeded for further characterization experiments.

\subsection{Physical stability of the selected aspasomal formulations}

As observed in Table 1, Formulations F2 and F3 exhibited good stability after 3 months storage at $4^{\circ} \mathrm{C}$, manifested by minor increase in the particle size, and a minor decrease in EE\%. Moreover, no significant change in the zeta potential values occurred after storage $(\mathrm{P}>0.05)$, further confirming the stability of aspasomes. The stability study was conducted at refrigeration rather than ambient conditions since the former is the standard storage protocol for vesicular systems (Nasr and Wahdan, 2019), to avoid the hydrolysis of lipids.

\subsection{Ex-vivo skin deposition of quercetin aspasomes}

The ex-vivo deposition of the quercetin loaded aspasomes in rat skin was investigated. Both formulations were able to deposit quercetin in the skin $(27.10 \% \pm 0.018)$ and $(40.93 \% \pm 0.022)$ for formulations F2 and F3 respectively, as shown in Figure 1. This could be ascribed to the film forming nature of vesicles, resulting in an occlusive effect and a decrease in the trans-epidermal water loss, therefore allowing more deposition of the drug (Jenning et al., 2000; Muller et al., 2007). In addition, it was reported by Lauchli et al., 2012 that neem oil exerts a semi-occlusive effect on the skin, causing a balanced moist environment, and hence increases deposition. The 
significantly lower deposition obtained with F2 compared to F3 may be ascribed to the smaller particle size of the latter, which allowed better accumulation in the outer skin layers (Puglia et al., 2006; Adib et al., 2016).

The cumulative percentage permeated of quercetin to the receptor compartment was less than $1 \%$ in both formulations; suggesting the topical rather than the transdermal traits of the quercetin loaded aspasomes. This low permeation rate of quercetin could be ascribed to its hydrophobic nature, as well as the hydrophobic nature of aspasomes (Tan et al., 2011; Jangde and Singh, 2016). Lastly, the presence of cholesterol may have accounted for the low quercetin permeation rates of both formulations, since it was reported by Nagarsenker and Londhe, 2003; Singh et al., 2014 and Briuglia et al., 2015 that cholesterol limits the movement of the relatively mobile hydrocarbon chains, and hence decreases the vesicular membrane permeability, and consequently the drug's permeation.

\subsection{Anti-oxidant assessment of quercetin aspasomes}

As evident in Figure 2, both formulations F2, F3 as well as the quercetin solution displayed high DPPH scavenging percentages at different concentrations. The aspasomal formulation F2 displayed significantly higher IC 50 value of $3.65 \mu \mathrm{g} / \mathrm{mL}$ compared to F3 which displayed $\mathrm{IC}_{50}$ value of $1.39 \mu \mathrm{g} / \mathrm{mL}(\mathrm{P}<0.05)$. The $\mathrm{IC}_{50}$ represents the concentration of quercetin causing $50 \%$ inhibition of the DPPH free radical, and hence F3 was considered more efficient in the free radical scavenging ability compared to F2, and its action was comparable to quercetin control solution, which displayed an $\mathrm{IC}_{50}$ of $1.52 \mu \mathrm{g} / \mathrm{mL}(\mathrm{P}>0.05)$, suggesting that $\mathrm{F} 3$ was able to preserve the antioxidant potential of quercetin. This came in accordance with Sessa et al., 2012 and Chen et al., 2015. The better antioxidant activity of formulation F3 compared to F2 can be correlated to the smaller particle size of the latter, as reported by Makanjuola, 2017. Moreover, the presence 
of neem oil which is known to contain many anti-oxidant molecules such as azadirachtin, ascorbic acid and a large number of phenolic compounds may have further contributed to its better free radical scavenging action (Olabinri et al., 2009; Hossain et al., 2014). Interestingly, the quercetin solution and aspasomal formulations displayed significantly lower $\mathrm{IC}_{50}$ values compared to the reference ascorbic acid, which showed an $\mathrm{IC}_{50}$ of $14.5 \mu \mathrm{g} / \mathrm{mL}$, suggesting that quercetin was a more potent antioxidant due to the presence of many hydroxyl groups in its structure (Rice-Evans et al., 1997; Pool et al.o, 2012).

Based on the combined effect of particle size, EE\%, physical stability, ex-vivo deposition and DPPH assays, F3 was chosen as the selected formulation for further characterization steps.

\subsection{Morphological characterization of the selected aspasomal formulation}

The quercetin aspasomal formulation F3 was visualized using TEM, which displayed the structure of well identified vesicles of sealed spherical core and coat nature, as shown in Figure 3.

\subsection{Assessment of the antibacterial potential of the selected aspasomal formulation}

The anti-bacterial effect was measured by calculating the average inhibition zone for quercetin and its aspasomal formulation F3, in which they displayed $8.25 \pm 2.08 \mathrm{~mm}$ and $15 \pm 1.53$ $\mathrm{mm}$ respectively. The larger inhibition zone encountered with the latter suggests that the presence of tea tree oil, neem oil, ascorbyl palmitate and the other aspasomes forming ingredients had potentiated the anti-acne activity of the formulation. Tea tree oil was reported to exhibit antibacterial action on P. acnes (Raman et al., 1995; Pazyar et al., 2013; Hammer, 2014; Wulansari et al., 2017) owing to the presence of terpinen-4-ol (Flores et al., 2011). Neem oil was also reported to exhibit an anti-bacterial activity against $P$. acnes (Alzohairy, 2016) owing to its content of various anti-bacterial ingredients used in acne treatment (Vijayan et al., 2013). 


\subsection{Safety of quercetin aspasomes on skin fibroblasts}

The neutral red assay is based on the ability of the viable cells to incorporate and bind the cationic neutral red dye after being treated with the test substance, by actively transporting the dye into the lysosomes (Repetto et al., 2008). When the viable cells are lysed, they release the dye, where the amount of the released dye is equivalent to the number of viable cells, and it can be measured spectrophotometrically at $540 \mathrm{~nm}$.

As shown in Figure 4, cells receiving no treatment displayed 100\% viability, while those receiving hydrogen peroxide as a positive control displayed significant death (26\% viability). The quercetin solution, and the aspasomal formulation F3 displayed high viability for the cells of $93.73 \pm 3.14$, and $92.47 \pm 3.99$ respectively $(\mathrm{P}<0.05)$. This may be attributed to the herbal nature of quercetin, and the use of highly safe ingredients in the preparation of the aspasomal formulation. The individual components of the formulations were all reported safe for topical use; Andersen, 1999; Lanigan, 1999 and Khan et al., 2016 reported that ascorbyl palmitate was considered safe, non-irritating for the skin, and showed no signs of sensitization in clinical studies. Cholesterol was also confirmed to be a non-toxic and a non-irritant ingredient when applied on the skin, where it is used as an emulsifier in the cosmetic products and considered safe to be used in skin products. As for the dicetyl phosphate, Ueoka and Moraes, 2018 reported its use as a surfactant and as an emulsifier as well in the cosmeceutical industry. Moreover, Pazyar et al., 2013; Hammer, 2014 and Rajkowska et al., 2014 reported that tea tree oil was considered non-toxic and safe to be applied on the skin. As for the neem oil, Aneesa and Gayathri, 2016 reported its use as an antiinflammatory, anti-allergic and an anti-bacterial oil when applied externally, and hence is considered safe when applied on to the skin. Quercetin itself was also reported to be used topically for wound healing and guarding against skin oxidative damage, which ensured its topical safety 
(Martin, 1996; Kumar et al., 2017). All this shows that both the quercetin solution, and the aspasomal formulation F3 are considered highly safe on the cellular level, hence nominating aspasomes for clinical testing.

\subsection{Clinical evaluation of quercetin aspasomes on acne patients}

After 2 months of applying the quercetin aspasomal formulation F3 on the acne lesions, the percentage reduction for the inflammatory lesions, the comedones, and the total acne lesions was calculated. As can be seen in Tables 2 and $\mathbf{3}$ and Figures 5 and 6, the aspasomal formulation F3 applied on acne lesions of the right side of the face displayed reduction percentages of $77.9 \%$, $11.8 \%$ and $55.3 \%$ for inflammatory lesions, comedones and total lesions respectively. There was a significant decrease in the count of inflammatory and total acne lesions $(\mathrm{P}<0.05)$ with no significant decrease in the count of comedones $(\mathrm{P}>0.05)$ compared to the left side receiving topical Panthenol $^{\circledR}$ cream, which displayed reduction percentages of $6.7 \%, 19.1 \%$ and $10.1 \%$ for inflammatory lesions, comedones and total lesions respectively. Aspasomes were more powerful on inflammatory lesions than on comedones, as evident from the significant higher percentage reduction for the former compared to the latter $(\mathrm{P}<0.05)$. Moreover, no local irritation, burning or dermatitis was observed during the treatment period ( 8 weeks) as reported by the patients treated with the aspasomal formulation F3. Patients reported that the formulation was devoid of greasiness and was light to apply. Moreover, they reported that it had retentive properties on the skin and was sufficiently acceptable.

Based on the aforementioned results, quercetin loaded aspasomes proved to be a promising nano-vesicular system for the treatment of different acne lesions. Their anti-inflammatory action as manifested by the significant reduction of the total lesion count (mainly the inflammatory lesions) could be ascribed to the use of tea tree oil, which exhibits anti-inflammatory properties as 
reported by Hart et al., 2000; Pazyar et al., 2013. Furthermore, Yadav and Rao, 2012; Aneesa and Gayathri, 2016 also reported that neem oil shows anti-inflammatory properties, which hence allows it to work better on inflammatory papules, rather than comedones. In addition, the presence of quercetin which also acts as an anti-inflammatory molecule majorly contributes to the antiinflammatory properties of F3 (Kumar et al., 2017). Moreover, Telang, 2013 reported that vitamin $\mathrm{C}$ was able to inhibit $\mathrm{NF}-\mathrm{kB}$, exerting an anti-inflammatory action, and hence, allowing the enhancement of the anti-inflammatory properties of F3 on the inflammatory papules rather than the comedones. Lastly, the amphipathic nature of the aspasomal vesicles in addition to their nanometer range allowed for their good skin interaction, and promotion of their therapeutic action (Hatem et al., 2018c).

\section{Conclusion}

As seen from all presented results, the preparation of vitamin C-based nano-vesicular system loaded with quercetin was proven to be successful in the treatment of acne vulgaris. This paves the way for the emergence of whole line of natural nanocosmeceutical delivery systems that can be successfully used in the treatment of other topical dermatological diseases.

\section{Acknowledgement}

The authors would like to acknowledge Dr. Simon Gould, and Dr. Ali Athab Al-kinan Discovery, Delivery and Patient Care (DDDPC) Theme, School of Life Sciences, Pharma Chemistry, Department of Pharmacy, Kingston University London, Kingston upon Thames, KT1 2EEfor their help in conducting the anti-bacterial and safety assays.

\section{References}


Abdelgawad, R., Nasr, M., Moftah, N.H., Hamza, M.Y., 2017. Phospholipid membrane tabulation using ceramide doping "cerosomes": characterization and clinical application in psoriasis treatment. Eur. J. Pharm. Sci. 101, 258-268.

Adib, Z.M., Ghanbarzadeh, S., Kouhsoltani, M., Khosroshahi, A.Y., Hamishehkar, H., 2016. The Effect of Particle Size on the Deposition of Solid Lipid Nanoparticles in Different Skin Layers: A Histological Study. Adv. Pharm. Bull. 6, 31-36.

Adityan, B., Kumari, R., Thappa, D.V., 2009. Scoring systems in acne vulgaris. Indian J. Dermatol. Venerol. Leprol. 75, 323-326.

Agiba, A.M., Nasr, M., Abdel-Hamid, S., Eldin, A.B., Geneidi, A.S., 2018. Enhancing the intestinal permeation of the chondroprotective nutraceuticals glucosamine sulphate and chondroitin sulphate using conventional and modified liposomes. Curr. Drug Deliv. 15, 907-916.

Alzohairy, M.A., 2016. Therapeutics Role of Azadirachtaindica (Neem) and their active constituents in diseases prevention and treatment. Evid. Based Complement. Alternat. Med. 2016, $1-11$.

Amer, S.S., Nasr, M., Mamdouh, W., Sammour, O., 2019. Insights on the use of nanocarriers for acne alleviation. Curr. Drug Deliv. 16, 18-25.

Andersen, F.A., 1999. Final Report on the safety assessment of ascorbyl palmitate, ascorbyldipalmitate, ascorbyl stearate, erythorbic acid, and sodium erythorbate. Int. J. Toxicol. 18, $1-26$.

Aneesa, N., Gayathri, J., 2016. Beneficial effects of neem oil-An updated review. J. Pharm. Sci. 
$8,756-758$.

Aref, N-E.M., Nasr, M., Osman, R., 2017. Novel heat-stable enterotoxin (STa) immunogen based on cationic nanoliposomes: Preparation, characterization, and immunization. J. Vaccin. Vaccination $8,1-8$.

Ashraf, O., Nasr, M., Nebsen, M., Said, A.M.A., Sammour, O., 2018. In vitro stabilization and in vivo improvement of ocular pharmacokinetics of the multi-therapeutic agent baicalin: Delineating the most suitable vesicular systems. Int. J. Pharm. 539, 83-94.

Ates, G., Vanhaecke, T., Rogiers, V., Rodrigues, R.M., 2017. Assaying cellular viability using the neutral red uptake assay. Methods Mol. Biol. 1601, 19-26.

Barakat, M.T., Moftah, N.H., El Khayyat, M.A.M., 2017. Significant reduction of inflammation and sebaceous glands size in acne vulgaris lesions after intense pulsed light treatment. Dermatol. Ther. 30, 1-5.

Bojar, R.A., Holland, K.T., 2004. Acne and Propionibacterium acnes. Clin. Dermatol. 22, 375379.

Briuglia, M-L., Rotella, C., McFarlane, A., Lamprou, D.A., 2015. Influence of cholesterol on liposome stability and on in vitro drug release. Drug Deliv. Transl. Res. 5, 231-242.

Bseiso, E.A., Nasr, M., Sammour, O., Abd El Gawad, N.A., 2015. Recent advances in topical formulation carriers of antifungal agents. Indian J. Dermatol. Venereol. Leprol. 81, 457-463. 
Bseiso, E.A., Nasr, M., Sammour, O.A., Abd El Gawad, N.A., 2016. Novel nail penetration enhancer containing vesicles "nPEVs" for treatment of onychomycosis. Drug Deliv. 23, 28132819.

Bsieso, E.A., Nasr, M., Moftah, N.H., Sammour, O.A., Abd El Gawad, N.A., 2015. Could nanovesicles containing a penetration enhancer clinically improve the therapeutic outcome in skin fungal diseases? Nanomedicine (Lond) 10, 2017-2031.

Calabro, A.R., Konsoula, R., Barile, F.A., 2008. Evaluation of in vitro cytotoxicity and paracellular permeability of intact monolayers with mouse embryonic stem cells. Toxicol. In Vitro 22, 12731284.

Chen, Y., Wu, Q., Zhang, Z., Yuan, L., Liu, X., Zhou, L., 2012. Preparation of curcumin-loaded liposomes and evaluation of their skin permeation and pharmacodynamics. Molecules 17, 59725987.

Chen, Y., Zhang, H., Yang, J., Sun, H., 2015. Improved antioxidant capacity of optimization of a self-microemulsifying drug delivery system for resveratrol. Molecules 20, 21167-21177.

Chibowski, E., Szcześ, A., 2016. Zeta potential and surface charge of DPPC and DOPC liposomes in the presence of PLC enzyme. Adsorption 22, 755-765.

Dali, P., Giugliano, E.R., Vellozzi, E.M., Smith, M.A., 2001. Susceptibilities of Propionibacterium acnes ophthalmic isolates to moxifloxacin. Antimicrob. Agents Chemother. 45, 2969-2970. 
Du, G., Hathout, R.M., Nasr, M., Nejadnik, M.R., Tu, J., Koning, R.I., Koster, A.J., Slutter, B., Kros, A., Jiskoot, W., Bouwstra, J.A., Monkare, J., 2017. Intradermal vaccination with hollow microneedles: A comparative study of various protein antigen and adjuvant encapsulated nanoparticles. J Control Release 266, 109-118.

El Zaafarany, G.M., Awad, G.A., Holayel, S.M., Mortada, N.D., 2010. Role of edge activators and surface charge in developing ultradeformable vesicles with enhanced skin delivery. Int. J. Pharm. 397, 164-172.

El-Kayal, M., Nasr, M., Elkheshen, S., Mortada, N., 2019. Colloidal (-)-epigallocatechin-3-gallate vesicular systems for prevention and treatment of skin cancer: A comprehensive experimental study with preclinical investigation. Eur. J. Pharm. Sci. 137, 104972.

Elmowafy, E., El-Gogary, R.I., Ragai, M.H., Nasr, M., 2019. Novel antipsoriatic fluidized spanlastic nanovesicles: In vitro physicochemical characterization, ex vivo cutaneous retention and exploratory clinical therapeutic activity. Int. J. Pharm. 568, 118556.

Enshaieh, S., Jooya, A., Siadat, A.H., Iraji, F., 2007. The efficacy of 5\% topical tea tree oil gel in mild to moderate acne vulgaris: a randomized, double-blind placebo-controlled study. Indian J. Dermatol. Venereol. Leprol. 73, 22-25.

Fabbrocini, G., Annunziata, M.C., D' Arco, V., De Vita, V., Lodi, G., Mauriello, M.C., Pastore, F., Monfrecola, G., 2010. Acne scars: pathogenesis, classification and treatment. Dermatol. Res. Pract. 2010, 1-13. 
Fadel, M., Kassab, K., Abd El Fadeel, D.A., Nasr, M., El Ghoubary, N.M., 2018. Comparative enhancement of curcumin cytotoxic photodynamic activity by nanoliposomes and gold nanoparticles with pharmacological appraisal in HepG2 cancer cells and Erlich solid tumor model. Drug Dev. Ind. Pharm. 44, 1809-1816.

Fadel, M., Samy, N., Nasr, M., Alyoussef, A.A., 2017. Topical colloidal indocyanine greenmediated photodynamic therapy for treatment of basal cell carcinoma. Pharm. Dev. Technol. 22, $545-550$.

Flores, F.C., Ribeiro, R.F., Ourique, A.F., Rolim, M.B., 2011. Nanostructured systems containing an essential oil: Protection against volatilization. Química Nova 34, 968-72.

Gopinath, D., Ravi, D., Rao, B.R., Apte, S.S., Renuka, D., Rambhau, D., 2004. Ascorbyl palmitate vesicles (Aspasomes): formation, characterization and applications. Int. J. Pharm. 271, 95-113.

Hammer, K.A., 2014. Treatment of acne with tea tree oil (melaleuca) products: A review of efficacy, tolerability and potential modes of action. Int. J. Antimicrob. Agents 45, 106-110.

Hart, P.H., Brand, C., Carson, C.F., Riley, T.V., Prager, R.H., Finlay-Jones, J.J., 2000. Terpinen4-ol, the main component of the essential oil of Melaleuca alternifolia (tea tree oil), suppresses inflammatory mediator production by activated human monocytes. Inflamm. Res. 49, 619-626.

Hatem, S., Nasr, M., Elkheshen, S.A., Geneidi, A.S., 2018a. Recent advances in antioxidant cosmeceutical topical delivery. Curr. Drug Deliv. 15, 953-964. 
Hatem, S., Nasr, M., Moftah, N.H., Ragai, M.H., Geneidi, A.S., Elkheshen, S.A., 2018b. Clinical cosmeceutical repurposing of melatonin in androgenic alopecia using nanostructured lipid carriers prepared with antioxidant oils. Expert Opin. Drug Deliv. 15, 927-935.

Hatem, S., Nasr, M., Moftah, N.H., Ragai, M.H., Geneidi, A.S., Elkheshen, S.A., 2018c. Melatonin vitamin C-based nanovesicles for treatment of androgenic alopecia: Design, characterization and clinical appraisal. Eur. J. Pharm. Sci. 15, 246-253.

Herman, A., Herman, A.P., 2014. Essential oils and their constituents as skin penetration enhancer for transdermal drug delivery: a review. J. Pharm. Pharmacol. 67, 473-485.

Hockley, K., Baxter, D., 1986. Use of 3T3 cell-neutral red uptake assay for irritants as an alternative to the rabbit (Draize) test. Food Chem. Toxicol. 24, 473-475.

Hossain, M.D., Sarwar, M.S., Dewan, S.M., Hossain, M.S., Shahid-Ud-Daula, A., Islam, M.S., 2014. Investigation of total phenolic content and antioxidant activities of Azadirachtaindica roots. Avicenna J. Phytomed. 4, 97-102.

Jangde, R., Singh, D., 2016. Preparation and optimization of quercetin-loaded liposomes for wound healing, using response surface methodology. Artif. Cells Nanomed. Biotechnol. 44, 635641.

Jenning, V., Schäfer-Korting, M., Gohla, S., 2000. Vitamin A-loaded solid lipid nanoparticles for topical use: drug release properties. J. Controlled Rel. 66, 115-126.

Jírová, D., Kejlová, K., Brabec, M., Bendová, H., Kolárová, H., 2003. The benefits of the 3T3 NRU test in the safety assessment of cosmetics: long-term experience from pre-marketing testing 
in Czech Republic. Toxicol. In Vitro 17, 791-796.

Khan, H., Akhtar, N., Khan, H.M.S., Arshad, A.I., Naeem, M., Sohail, M., Ali, A., Rasool, F., Nawaz, Z., 2016. Synergistic effects of ascorbyl palmitate and sodium ascorbyl phosphate loaded in multiple emulsions on facial skin melanin and erythema content. Biomed. Res. 27, 570-576.

Kirby, C., Clarke, J., Gregoriadis, G., 1980. Cholesterol content of small unilamellar liposomes controls phospholipid loss to high density lipoproteins in the presence of serum. FEBS Lett. 111, $324-328$.

Kishishita, M., Ushijima, T., Ozaki, Y., Ito, Y., 1980. New medium for isolating Propionibacteria and its application to assay of normal flora of human facial skin. Appl. Environ. Microbiol. 40, 1100-1105.

Kumar, G.S., Jayaveera, K.N., Kumar, C.K., Sanjay, U.P., Swamy, B.M., Kumar, D.V., 2007. Antimicrobial effects of Indian medicinal plants against acne-inducing bacteria. Trop. J. Pharm. Res. 6, 717-723.

Kumar, R., Vijayalakshmi, S., Nadanasabapathi, S., 2017. Health benefits of quercetin. Def. Life Sci. J. 2, 142-151.

Kumari, A., Yadav, S.K., Pakade, Y.B., Singh, B., Yadav, S.C., 2010. Development of biodegradable nanoparticles for delivery of quercetin. Colloids Surf. B Biointerfaces 80, 184-192.

Lanigan, R.S., 1999. Final report on the safety assessment of ascorbyl palmitate, ascorbyldipalmitate, ascorbyl stearate, erythorbic acid, and sodium erythorbate. Int. J. Toxicol. 18, $1-26$. 
Lasarow, R.M., Isseroff, R.R., Gomez, E.C., 1992. Quantitative in vitro assessment of phototoxicity by a fibroblast-neutral red assay. J. Investig. Dermatol. 98, 725-729.

Läuchli, S., Hafner, J., Wehrmann, C., French, L.E., Hunziker, T., 2012. Post-surgical scalp wounds with exposed bone treated with a plant-derived wound therapeutic. J. Wound Care 21, $228-233$.

Lee, S-C., Lee, K-E., Kim, J-J., Lim, S.H., 2005.The effect of cholesterol in the liposome bilayer on the stabilization of incorporated retinol. J. Liposome Res. 15, 157-166.

Maciel, R.M., Costa, M.M., Martins, D.B., Franca, R.T., Schmatz, R., Graca, D.L., Duarte, M.M., Danesi, C.C., Mazzanti, C.M., Schetinger, M.R., Paim, F.C., Palma, H.E., Abdala, F.H., Stefanello, N., Zimpel, C.K., Felin, D.V., Lopes, S.T., 2013. Antioxidant and anti-inflammatory effects of quercetin in functional and morphological alterations in streptozotocin-induced diabetic rats. Res. Vet. Sci. 95, 389-397.

Magarkar, A., Dhawan, V., Kallinteri, P., Viitala, T., El mowafy, M., Róg, T., Bunker, A., 2014. Cholesterol level affects surface charge of lipid membranes in saline solution. Sci. Rep. 4, 1-5.

Makanjuola, S.A., 2017. Influence of particle size and extraction solvent on antioxidant properties of extracts of tea, ginger, and tea-ginger blend. Food Sci. Nutr. 5, 1179-1185.

Martin, A., 1996. The use of antioxidants in healing. Dermatol. Surg. 22, 156-160.

Meves, A., Stock, S.N., Beyerle, A., Pittelkow, M.R., Peus, D., 2002. Vitamin C derivative ascorbyl palmitate promotes ultraviolet-B-induced lipid peroxidation and cytotoxicity in 
keratinocytes. J. Invest. Dermatol. 119, 1103-1108.

Mills, O.H., Criscito, M.C., Schlesinger, T.E., Verdicchio, R., Szoke, E., 2016. Addressing free radical oxidation in Acne Vulgaris. J. Clin. Aesthet. Dermatol. 9, 25-30.

Moribe, K., Maruyama, K., Iwatsuru, M., 1999. Encapsulation characteristics of nystatin in liposomes: effects of cholesterol and polyethylene glycol derivatives. Int. J. Pharm. 188, 193-202.

Morilla, M.J., Benavidez, P., Lopez, M.O., Bakas, L., Romero, E.L., 2002. Development and in vitro characterisation of a benznidazole liposomal formulation. Int. J. Pharm. 249, 89-99.

Morris, G.E., 1954. Use of vitamin C in acne vulgaris. A.M.A. Arch. Derm. Syphilol. 70, 363-364.

Mouez, M.A., Nasr, M., Abdel-Mottaleb, M., Geneidi, A.S., Mansour, S., 2016. Composite chitosan-transfersomal vesicles for improved transnasal permeation and bioavailability of verapamil. Int. J. Biol. Macromol. 93, 591-599.

Muller, R.H., Peterson, R.D., Hommoss, A., Pardeike, J., 2007. Nanostructured lipid carriers (NLC) in cosmetic dermal products. Adv. Drug Deliv. Rev. 59, 522-530.

Nagarsenker, M., Londhe, V., 2003. Preparation and evaluation of a liposomal formulation of sodium cromoglicate. Int. J. Pharm. 251, 49-56.

Najafi-Taher, R., Amani, A., 2017. Nanoemulsions: colloidal topical delivery systems for antiacne agents- A Mini-Review. Nanomed. Res. J. 2, 49-56.

Nasr, M., Abdel-Hamid, S., 2016. Optimizing the dermal accumulation of a tazarotene microemulsion using skin deposition. Drug Dev. Ind. Pharm. 42, 636-643. 
Nasr, M., Abdel-Hamid, S., Moftah, N.H., Fadel, M., Alyoussef, A.A., 2017. Jojoba oil soft colloidal nanocarrier of a synthetic retinoid: preparation, characterization and clinical efficacy in psoriatic patients. Curr. Drug Deliv.14, 426-32.

Nasr, M., Karandikar, H., Abdel-Aziz, R.T.A., Moftah, N., Paradkar, A., 2018. Novel nicotinamide skin-adhesive hot melt extrudates for treatment of acne. Expert Opin. Drug Deliv. $15,1165-73$.

Nasr, M., Mansour, S., Mortada, N.D., ElShamy, A.A., 2008. Vesicular aceclofenac systems: a comparative study between liposomes and niosomes. J. Microencapsulation 25, 499-512.

Nasr, M., Taha, I., Hathout, R.M., 2013. Suitability of liposomal carriers for systemic delivery of risedronate using the pulmonary route. Drug Deliv. 20, 311-318.

Nasr, M., Wahdan, S.A., 2019. Neuroprotective effects of novel nanosystems simultaneously loaded with vinpocetine and piracetam after intranasal administration. Life Sci. 226, 117-129.

NilFroushzadeh, M.A., Siadat, A.H., Baradaran, E.H., Moradi, S., 2009. Clindamycin lotion alone versus combination lotion of clindamycin phosphate plus tretinoin versus combination lotion of clindamycin phosphate plus salicylic acid in the topical treatment of mild to moderate acne vulgaris: A randomized control trial. Indian J. Dermatol. Venerol. Leprol. 75, 279-282.

Olabinri, B.M., Adebisi, J.A., Odesomi, O.F., Olabinri, P.F., Adeleke, G.E., 2009. Experimental classification of the antioxidant capacity of the leaf, stem and root barks of Magnifera indica and 
Azadirachta indica. Afr. J. Biotechnol. 8, 2968-2972.

Owen, M.C., Kulig, W., Rog, T., Vattulainen, L., Strodel, B., 2018. Cholesterol Protects the Oxidized Lipid Bilayer from Water Injury: An All-Atom Molecular Dynamics Study. J. Membr. Biol. 251, 521-534.

Pazyar, N., Yaghoobi, R., Bagherani, N., Kazerouni, A., 2013. A review of applications of tea tree oil in dermatology. Int. J. Dermatol. 52, 784-790.

Peirce, R.J., Buker, W.H., Brody, S.L., 1959. Hesper-C in acne. Ohio Med. J. 55:59.

Perry, A.L., Lambert, P.A., 2006. Propionibacterium acnes. Lett. Appl. Microbiol. 42, 185-188.

Pool, H., Quintanar, D., Figueroa, J.de Dios., Mano, C.M., Bechara, J.E.H., Godínez, L.A., Mendoza, S., 2012. Antioxidant effects of quercetin and catechin encapsulated into PLGA nanoparticles. J. Nanomater. 2012, 1-12.

Puglia, C., Filosa, R., Peduto, A., de Caprariis, P., Rizza, L., Bonina, F., Blasi, P., 2006. Evaluation of alternative strategies to optimize ketorolac transdermal delivery. AAPS J. 7, E61-E69.

Rajkowska, K., Kunicka-Styczyńska, A., Maroszyńska, M., Dąbrowska, M., 2014. The effect of thyme and tea tree oils on morphology and metabolism of Candida albicans. Acta Biochim. Pol. 61, 305-310.

Raman, A., Weir, U., Bloomfield, S.F., 1995. Antimicrobial effects of tea-tree oil and its major components on Staphylococcus aureus, Staph. epidermidis and Propionibacterium acnes. Lett. Appl. Microbiol. 21, 242-245. 
Repetto, G., del Pesso, A., Zurita, J.L., 2008. Neutral red uptake assay for the estimation of cell viability/cytotoxicity. Nat. Protoc. 3, 1125-1131.

Rice-Evans, C.A., Miller, N.J., Paganga, G., 1997. Antioxidant properties of phenolic compounds. Trends Plant Sci. 2, 152-159.

Rothwell, J.A., Day, A.J., Morgan, M.R., 2005. Experimental determination of octanol-water partition coefficients of quercetin and related flavonoids. J. Agr. Food Chem. 53, 4355-4360.

Saini, R.K., Shetty, N.P., Prakash, M., Giridhar, P., 2014. Effect of dehydration methods on retention of carotenoids, tocopherols, ascorbic acid and antioxidant activity in Moringa oleifera leaves and preparation of a RTE product. J. Food Sci. Technol. 51, 2176-2182.

Sessa, M., Casazza, A.A., Perego, P., Tsao, R., Ferrari, G., Donsi, F., 2012. Exploitation of polyphenolic extracts from grape marcas natural antioxidants by encapsulation in lipid-based nanodelivery system. Food Bioprocess Tech. 6, 2609-2620.

Shaaban, M., Nasr, M., Tawfik, A.A., Fadel, M., Sammour, O., 2019. Novel bergamot oil nanospanlastics combined with PUVB therapy as a clinically translatable approach for vitiligo treatment. Drug Deliv. Transl. Res. 9, 1106-1116.

Sharma, A., Sharma, U.S., 1997. Liposomes in drug delivery: progress and limitations. Int. J. Pharm. 154, 123-140.

Singh, A., Vengurlekar, P., Rathod, S., 2014. Design, development, and characterization of liposomal neem gel. Int. J. Pharm. Sci. Res. 5, 140-148. 
Sinico, C., Manconi, M., Peppi, M., Lai, F., Valenti, D., Fadda, A.M., 2005. Liposomes as carriers for dermal delivery of tretinoin: in vitro evaluation of drug permeation and vesicle-skin interaction. J. Control. Release 103, 123-136.

Strauss, J.S., Krowchuk, D.P., Leyden, J.J., Lucky, A.W., Shalita, A.R., Siegfried, E.C., Thiboutot, D.M., Van Voorhees, A.S., Beutner, K.A., Sieck, C.K., Bhushan, R., 2007. Guidelines of care for acne vulgaris management. J. Am. Acad. Dermatol. 56, 651-663.

Tally, F.P., Goldin, B.R., Sullivan, N., Johnston, J., Gorbach, S.L., 1978. Antimicrobial activity of metronidazole. Antimicrob. Agents Chemother. 13, 460-465.

Tan, Q., Liu, W., Guo, C., Zhai, G., 2011. Preparation and evaluation of quercetin-loaded lecithinchitosan nanoparticles for topical delivery. Int. J. Nanomedicine 6, 1621-1630.

Telang, P.S., 2013. Vitamin C in dermatology. Indian Dermatol. Online J. 4, 143-146.

Titus, S., Hodge, J., 2012. Diagnosis and treatment of acne. Am. Fam. Physician 86, 734-740.

Ueoka, A.R., Moraes, C.A.P., 2018. Development and stability evaluation of liquid crystal-based formulations containing glycolic plant extracts and nano-actives. Cosmetics 5, 1-7.

Ulbricht, C., Seamon, E., Windsor, R.C., Armbruester, N., Bryan, J.K., Costa, D., Giese, N., Gruenwald, J., Iovin, R., Isaac, R., Serrano, J.M.G., Tanguay-Colucci, S., Weissner, W., Yoon, H., Zhang, J., 2011. An Evidence-based systematic review of cinnamon (cinnamomum spp.) by the natural standard research collaboration. J. Diet. Suppl. 8, 378-454.

Vijayan, V., Aafreen, S., Sakthivel, S., Reddy, K.R., 2013. Formulation and characterization of 
solid lipid nanoparticles loaded neem oil for topical treatment of acne. J. Acute Dis. 2, 282-286.

Villasmil-Sánchez, S., Drhimeur, W., Ospino, S.C.S., Rabasco Alvarez, A.M., GonzálezRodríguez, M.L., 2010. Positively and negatively charged liposomes as carriers for transdermal delivery of sumatriptan: in vitro characterization. Drug Dev. Ind. Pharm. 36, 666-675.

Wulansari, A., Jufri, M., Budianti, A., 2017. Studies on the formulation, physical stability, and in vitro antibacterial activity of tea tree oil (Melaleuca Alternifolia) nanoemulsion gel. Int. J. App. Pharm. 9, 135-139.

Yadav, K.C.H., Rao, Y.V., 2012. Evaluation of topical anti-inflammatory effect of azadirachtaindica leaf extract. Int. Res. J. Pharm. App. Sci. 2, 60-64.

\section{Figure legends}

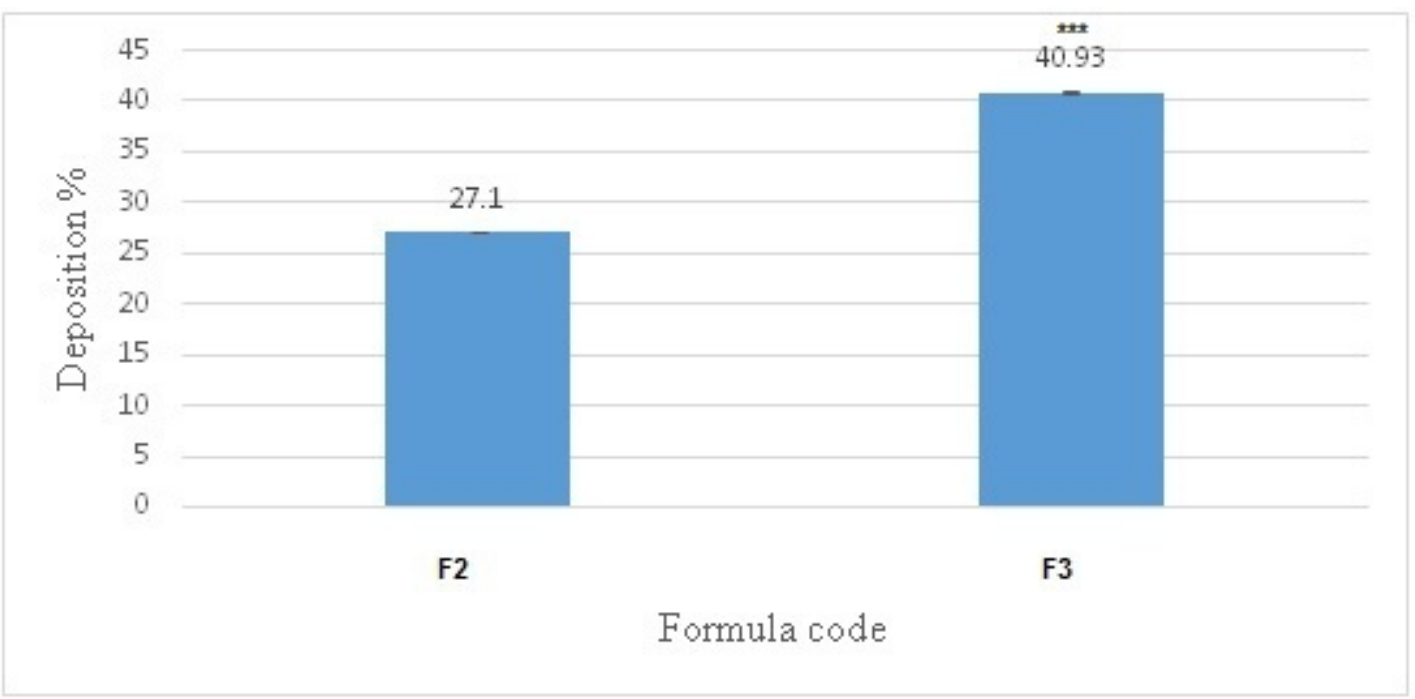


Figure 1: Skin deposition percentage of quercetin from formulations F2 and F3, displaying significantly higher deposition for the latter $(* * * \mathrm{P}<0.0001)$.



Figure 2: DPPH free radical scavenging effects of quercetin vesicular formulations F2 and F3 compared to quercetin control solution and ascorbic acid reference (Mean \pm S.D), showing the ability of formulation F3 to preserve the antioxidant potential of quercetin. 


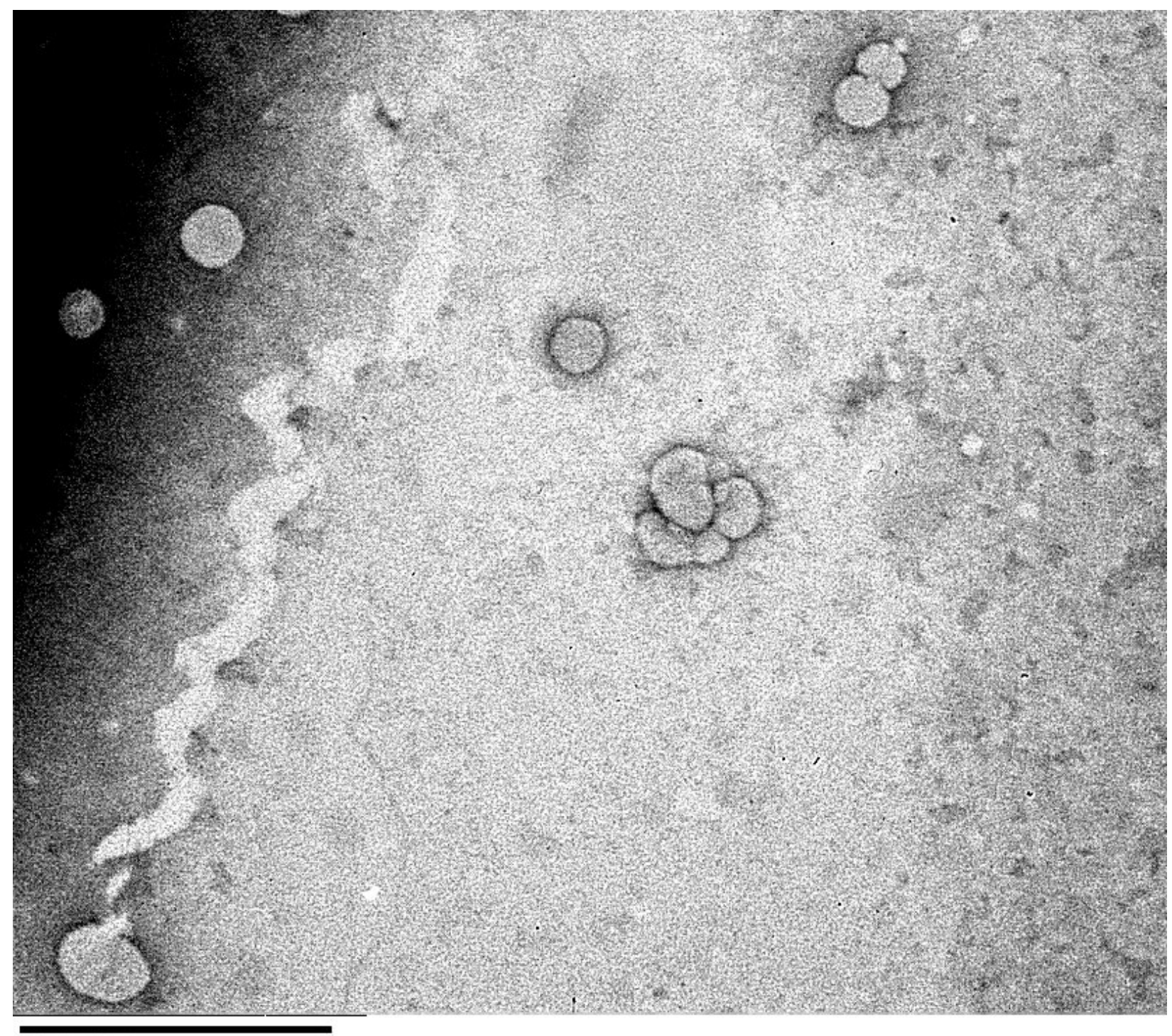

$500 \mathrm{~nm}$

TEM Mag $=60000 x$

Figure 3: TEM micrograph of F3 at a magnification of $60000 \mathrm{X}$ displaying the spherical nature of the aspasomal vesicles. 


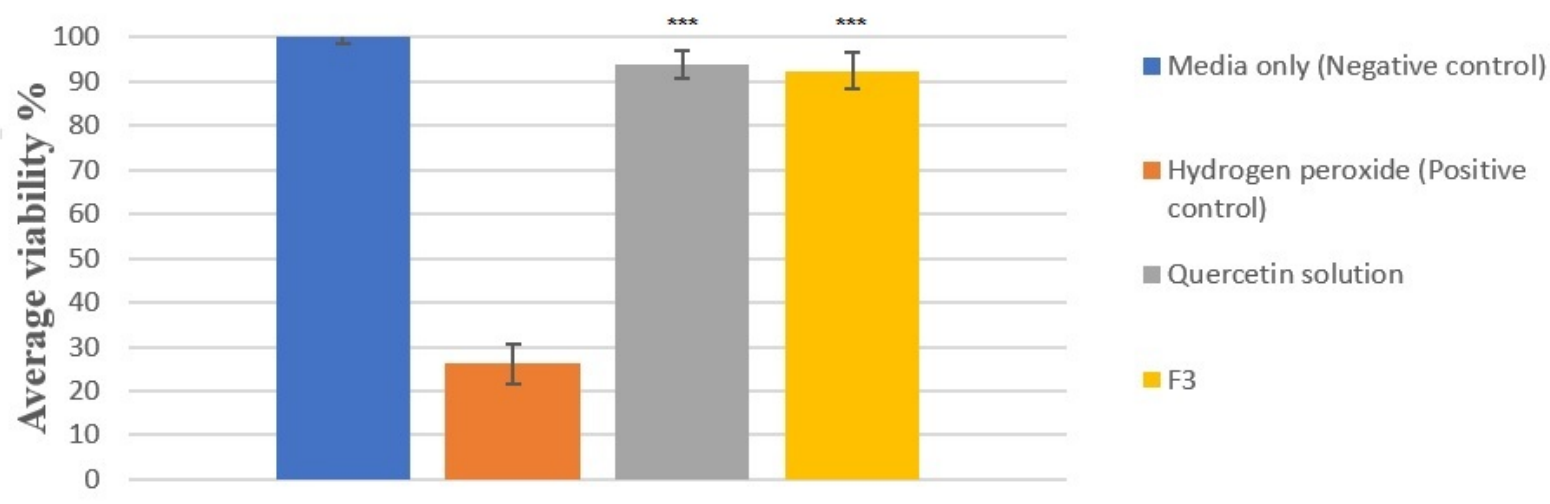

Figure 4: The average viability percentages of the cells when treated with different samples (the selected formulation F3 in comparison to the media as negative control, hydrogen peroxide as positive control and the quercetin solution (*** significantly higher viability from positive control $(\mathrm{P}<0.0001)$, and insignificantly different from each other $\mathrm{P}>0.05)$. 


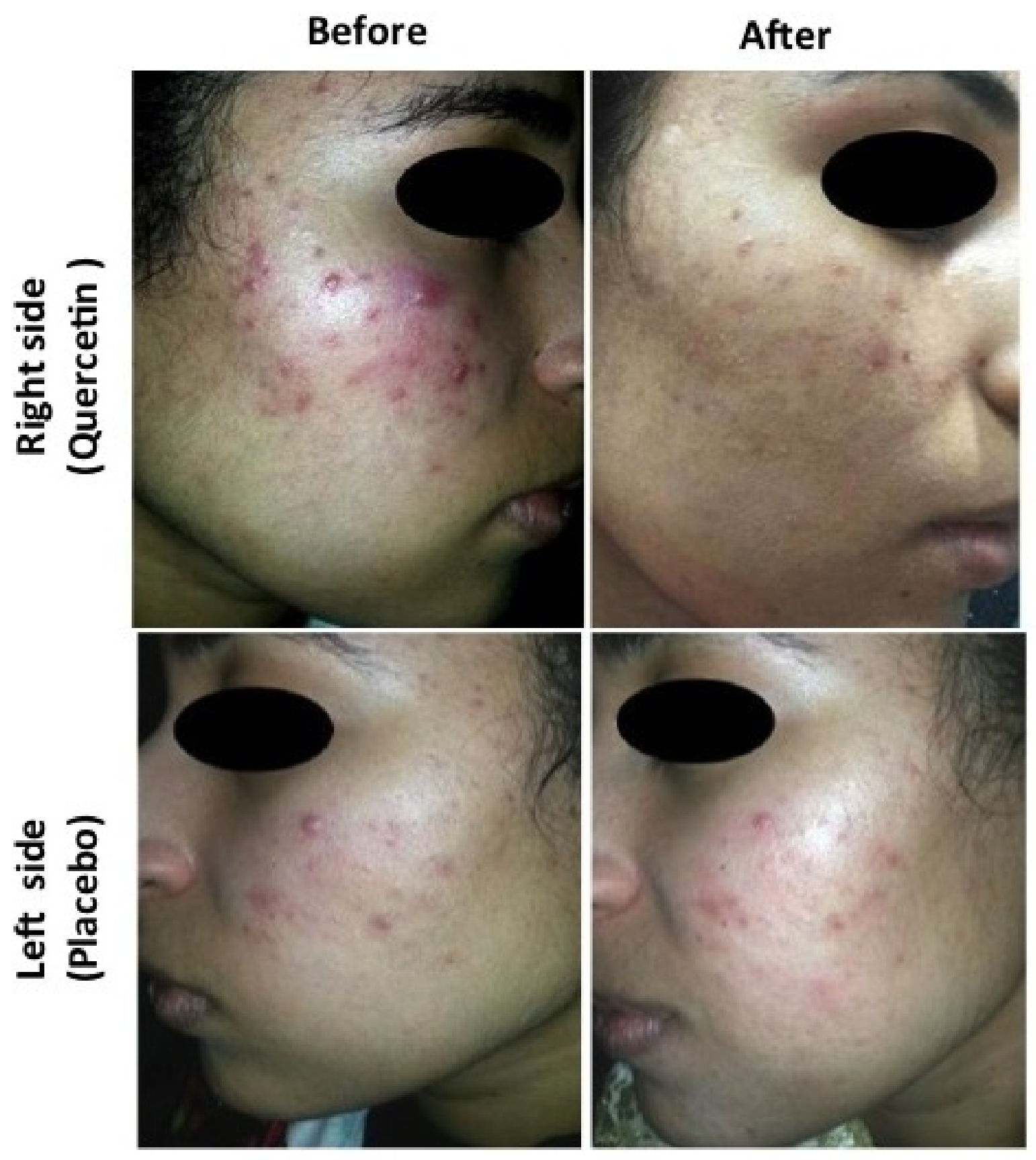

Figure 5: Representative patient receiving aspasomal formulation F3 on the right side and Panthenol ${ }^{\circledR}$ cream on the left side, showing better anti-acne effect of the former manifested by better reduction in the number of acne lesions. 


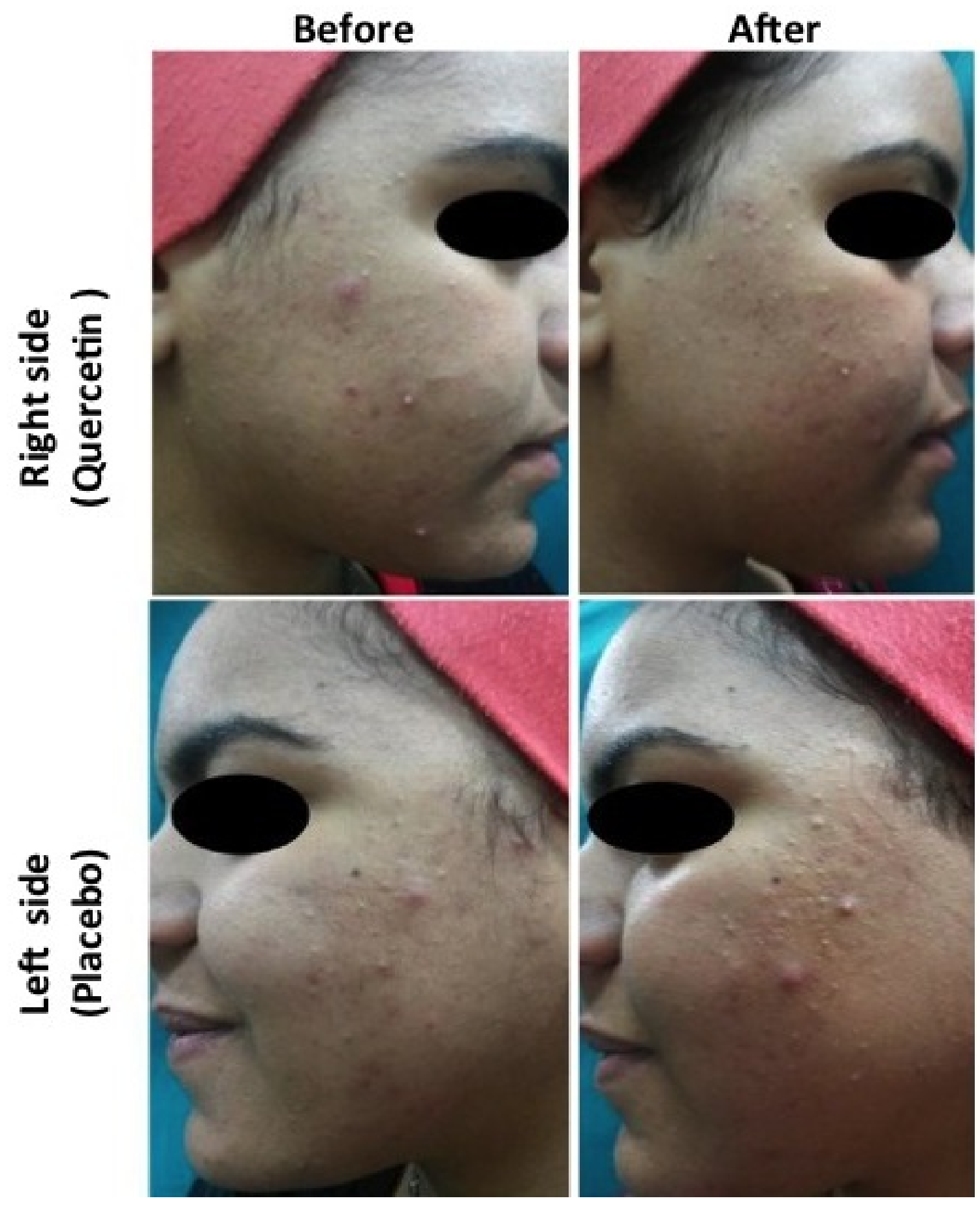

Figure 6: Representative patient receiving aspasomal formulation F3 on the right side and Panthenol ${ }^{\circledR}$ cream on the left side, showing better anti-acne effect of the former manifested by better reduction in the number of acne lesions. 
Table 1: Characterization of the prepared quercetin aspasomal formulations

\begin{tabular}{|c|c|c|c|c|c|c|c|}
\hline $\begin{array}{c}\text { Formula } \\
\text { code* }\end{array}$ & Oil & $\begin{array}{c}\text { Particle } \\
\text { size } \\
(\mathbf{n m}) \\
\text { Mean } \pm \text { S.D. } \\
\text { (freshly } \\
\text { prepared) }\end{array}$ & $\begin{array}{c}\text { Particle size } \\
(\text { nm) } \\
\text { Mean } \pm \text { S.D. } \\
\text { (after } \\
\text { storage) }\end{array}$ & $\begin{array}{c}\text { Zeta } \\
\text { potential } \\
(\mathbf{m V}) \\
\text { Mean } \pm \text { S.D. } \\
\text { (freshly } \\
\text { prepared) }\end{array}$ & $\begin{array}{c}\text { Zeta } \\
\text { potential } \\
(\mathbf{m V}) \\
\text { Mean } \pm \text { S.D. } \\
\text { (after } \\
\text { storage) }\end{array}$ & $\begin{array}{c}\text { EE \% } \\
\text { Mean } \pm \text { S.D. } \\
\text { (freshly } \\
\text { prepared) } \\
\text { and content } \\
\text { (mg/g of } \\
\text { formulation) }\end{array}$ & $\begin{array}{c}\text { EE \% } \\
\text { Mean } \pm \text { S.D. } \\
\text { (after } \\
\text { storage) } \\
\text { and content } \\
\text { (mg/g of } \\
\text { formulation) }\end{array}$ \\
\hline F1 & $\begin{array}{c}10 \mu \mathrm{L} \\
\text { neem oil }\end{array}$ & $184 \pm 7$ & N.D. & $-100 \pm 13$ & N.D. & $\begin{array}{c}96 \pm 0.1 \\
(0.96 \mathrm{mg} / \mathrm{g} \text { of } \\
\text { formulation })\end{array}$ & N.D. \\
\hline $\mathrm{F} 2$ & $\begin{array}{c}10 \mu \mathrm{L} \text { tea } \\
\text { tree oil }\end{array}$ & $182 \pm 5$ & $206 \pm 3$ & $-87 \pm 7$ & $-84 \pm 4$ & $\begin{array}{c}100 \pm 0.01 \\
(1 \mathrm{mg} / \mathrm{g} \text { of } \\
\text { formulation) }\end{array}$ & $\begin{array}{c}93 \pm 0.02 \\
(0.93 \mathrm{mg} / \mathrm{g} \\
\text { of } \\
\text { formulation) }\end{array}$ \\
\hline F3 & $\begin{array}{c}10 \mu \mathrm{L} \\
\text { neem oil } \\
+10 \mu \mathrm{L} \\
\text { tea tree oil }\end{array}$ & $125 \pm 3$ & $142 \pm 2$ & $-80 \pm 2$ & $-77 \pm 2$ & $\begin{array}{c}100 \pm 0.01 \\
(1 \mathrm{mg} / \mathrm{g} \text { of } \\
\text { formulation) }\end{array}$ & $\begin{array}{c}91 \pm 0.01 \\
(0.91 \mathrm{mg} / \mathrm{g} \\
\text { of } \\
\text { formulation })\end{array}$ \\
\hline
\end{tabular}

* All formulae were rehydrated with $10 \mathrm{ml}$ phosphate buffer (PB pH 7.4), and contained $10 \mathrm{mg}$ quercetin 
Table 2: The percentage reduction for the inflammatory lesions, comedones, and the total lesions for patients after application of aspasomal formulation $F 3$ on the right side of the face

\begin{tabular}{|c|c|c|c|c|c|c|c|c|c|}
\hline \multicolumn{10}{|c|}{ Right side } \\
\hline $\begin{array}{l}\text { Patient } \\
\text { number }\end{array}$ & Inf.B & Inf.A & $\begin{array}{c}\% \\
\text { reduction* }\end{array}$ & Com.B & Com.A & $\begin{array}{c}\% \\
\text { reduction* }\end{array}$ & Tot.B & Tot.A & $\begin{array}{c}\% \\
\text { reduction* }\end{array}$ \\
\hline 1 & 13 & 3 & 76.9 & 11 & 10 & 9.1 & 24 & 13 & 45.8 \\
\hline 2 & 17 & 2 & 88.2 & 8 & 7 & 12.5 & 25 & 9 & 64.0 \\
\hline 3 & 15 & 3 & 80.0 & 2 & 2 & 0.0 & 17 & 5 & 70.6 \\
\hline 4 & 13 & 2 & 84.6 & 9 & 8 & 11.1 & 22 & 10 & 54.5 \\
\hline 5 & 14 & 2 & 85.7 & 11 & 9 & 18.2 & 25 & 11 & 56.0 \\
\hline 6 & 8 & 1 & 87.5 & 4 & 4 & 0.0 & 12 & 5 & 58.3 \\
\hline 7 & 9 & 1 & 88.9 & 10 & 9 & 10.0 & 19 & 10 & 47.4 \\
\hline 8 & 12 & 3 & 75.0 & 7 & 7 & 0.0 & 19 & 10 & 47.4 \\
\hline 9 & 10 & 3 & 70.0 & 6 & 4 & 33.3 & 16 & 7 & 56.3 \\
\hline 10 & 11 & 2 & 81.8 & 8 & 6 & 25.0 & 19 & 8 & 57.9 \\
\hline 11 & 13 & 2 & 84.6 & 4 & 3 & 25.0 & 17 & 5 & 70.6 \\
\hline 12 & 14 & 2 & 85.7 & 7 & 7 & 0.0 & 21 & 9 & 57.1 \\
\hline 13 & 17 & 2 & 88.2 & 9 & 9 & 0.0 & 26 & 11 & 57.7 \\
\hline 14 & 11 & 4 & 63.6 & 2 & 2 & 0.0 & 13 & 6 & 53.8 \\
\hline 15 & 12 & 2 & 83.3 & 8 & 7 & 12.5 & 20 & 9 & 55.0 \\
\hline 16 & 13 & 6 & 53.8 & 11 & 11 & 0.0 & 24 & 17 & 29.2 \\
\hline 17 & 11 & 5 & 54.5 & 10 & 8 & 20.0 & 21 & 13 & 38.1 \\
\hline 18 & 12 & 3 & 75.0 & 5 & 5 & 0.0 & 17 & 8 & 52.9 \\
\hline 19 & 15 & 2 & 86.7 & 5 & 2 & 60.0 & 20 & 4 & 80.0 \\
\hline 20 & 14 & 5 & 64.3 & 3 & 3 & 0.0 & 17 & 8 & 52.9 \\
\hline & \multicolumn{2}{|c|}{$\begin{array}{l}\text { Average \% } \\
\text { reduction }\end{array}$} & $77.9 \pm 11.1$ & \multicolumn{2}{|c|}{$\begin{array}{c}\text { Average \% } \\
\text { reduction }\end{array}$} & $11.8 \pm 15.4$ & \multicolumn{2}{|c|}{$\begin{array}{l}\text { Average } \% \\
\text { reduction }\end{array}$} & $55.3 \pm 11.2$ \\
\hline
\end{tabular}

* Percent reduction was calculated by subtracting the number of lesions after administration $A$ from the number of lesions before administration $B$ and dividing by the latter

Inf. : Inflammatory lesions

Com. :Comedones

Tot. : Total number of inflammatory lesions and comedones 
Table 3: The percentage reduction for the inflammatory lesions, comedones, and the total lesions for patients after application of Panthenol ${ }^{\circledR}$ cream as placebo on the left side of the face

\begin{tabular}{|c|c|c|c|c|c|c|c|c|c|}
\hline \multicolumn{10}{|c|}{ Left side } \\
\hline $\begin{array}{l}\text { Patient } \\
\text { number }\end{array}$ & Inf.B & Inf.A & $\begin{array}{c}\% \\
\text { reduction* }\end{array}$ & Com.B & Com.A & $\begin{array}{c}\% \\
\text { reduction* }\end{array}$ & Tot.B & Tot.A & $\begin{array}{c}\% \\
\text { reduction* }\end{array}$ \\
\hline 1 & 14 & 13 & 7.1 & 10 & 9 & 10.0 & 24 & 22 & 8.3 \\
\hline 2 & 18 & 17 & 5.6 & 7 & 6 & 14.3 & 25 & 23 & 8.0 \\
\hline 3 & 16 & 17 & -6.3 & 3 & 3 & 0.0 & 19 & 20 & -5.3 \\
\hline 4 & 15 & 14 & 6.7 & 7 & 6 & 14.3 & 22 & 20 & 9.1 \\
\hline 5 & 14 & 13 & 7.1 & 10 & 8 & 20.0 & 24 & 21 & 12.5 \\
\hline 6 & 9 & 9 & 0.0 & 4 & 4 & 0.0 & 13 & 13 & 0.0 \\
\hline 7 & 11 & 9 & 18.2 & 11 & 9 & 18.2 & 22 & 18 & 18.2 \\
\hline 8 & 13 & 11 & 15.4 & 8 & 7 & 12.5 & 21 & 18 & 14.3 \\
\hline 9 & 11 & 11 & 0.0 & 7 & 6 & 14.3 & 18 & 17 & 5.6 \\
\hline 10 & 13 & 12 & 7.7 & 7 & 5 & 28.6 & 20 & 17 & 15.0 \\
\hline 11 & 12 & 11 & 8.3 & 3 & 2 & 33.3 & 15 & 13 & 13.3 \\
\hline 12 & 12 & 10 & 16.7 & 8 & 5 & 37.5 & 20 & 15 & 25.0 \\
\hline 13 & 15 & 14 & 6.7 & 10 & 9 & 10.0 & 25 & 23 & 8.0 \\
\hline 14 & 10 & 10 & 0.0 & 3 & 3 & 0.0 & 13 & 13 & 0.0 \\
\hline 15 & 15 & 13 & 13.3 & 7 & 6 & 14.3 & 22 & 19 & 13.6 \\
\hline 16 & 14 & 11 & 21.4 & 10 & 10 & 0.0 & 24 & 21 & 12.5 \\
\hline 17 & 11 & 11 & 0.0 & 11 & 8 & 27.3 & 22 & 19 & 13.6 \\
\hline 18 & 14 & 14 & 0.0 & 5 & 4 & 20.0 & 19 & 18 & 5.3 \\
\hline 19 & 17 & 16 & 5.9 & 5 & 3 & 40.0 & 22 & 19 & 13.6 \\
\hline 20 & 14 & 14 & 0.0 & 3 & 1 & 66.7 & 17 & 15 & 11.8 \\
\hline & \multicolumn{2}{|c|}{$\begin{array}{l}\text { Average } \% \\
\text { reduction }\end{array}$} & $6.7 \pm 7.3$ & \multicolumn{2}{|c|}{$\begin{array}{l}\text { Average } \% \\
\text { reduction }\end{array}$} & $19.1 \pm 16.5$ & \multicolumn{2}{|c|}{$\begin{array}{l}\text { Average \% } \\
\text { reduction }\end{array}$} & $10.1 \pm 6.8$ \\
\hline
\end{tabular}

* Percent reduction was calculated by subtracting the number of lesions after administration $A$ from the number of lesions before administration $B$ and dividing by the latter

Inf. : Inflammatory lesions

Com. :Comedones

Tot. : Total number of inflammatory lesions and comedones 
